\title{
ON THE ORIGIN OF RADIATION GROWTH OF HCP CRYSTALS
}

November 2011

\section{S. I. GOLUBOV}

Oak Ridge National Laboratory

A. V. BARASHEV

Oak Ridge National Laboratory

University of Tennessee

R. E. STOLLER

Oak Ridge National Laboratory 


\section{DOCUMENT AVAILABILTY}

Reports produced after January 1, 1996, are generally available free via the U.S. Department of Energy (DOE) Information Bridge.

Web site $\mathrm{http}: / / \mathrm{www}$.osti.gov/bridge

Reports produced before January 1, 1996, may be purchased by members of the public from the following source.

National Technical Information Service

5285 Port Royal Road

Springfield, VA 22161

Telephone 703-605-6000 (1-800-553-6847)

TDD 703-487-4639

Fax 703-605-6900

E-mailinfo@ntis.gov

Web site http://www.ntis.gov/support/ordernowabout.htm

Reports are available to DOE employees, DOE contractors, Energy Technology Data Exchange (ETDE)

representatives, and International Nuclear Information System (INIS) representatives from the following source. Office of Scientific and Technical Information

P.O. Box 62

Oak Ridge, TN 37831

Telephone 865-576-8401

Fax 865-576-5728

E-mail reports@osti.gov

Web site http://www.osti.gov/contact.html

This report was prepared as an account of work sponsored by an agency of the United States Government. Neither the United States Government nor any agency thereof, nor any of their employees, makes any warranty, express or implied, or assumes any legal liability or responsibility for the accuracy, completeness, or usefulness of any information, apparatus, product, or process disclosed, or represents that its use would not infringe privately owned rights. Reference herein to any specific commercial product, process, or service by trade name, trademark, manufacturer, or otherwise, does not necessarily constitute or imply its endorsement, recommendation, or favoring by the United States Government or any agency thereof. The views and opinions of authors expressed herein do not necessarily state or reflect those of the United States Government or any agency thereof 


\title{
ON THE ORIGIN OF RADIATION GROWTH OF HCP CRYSTALS
}

\author{
S. I. GOLUBOV \\ Oak Ridge National Laboratory \\ A. V. BARASHEV \\ Oak Ridge National Laboratory \\ The University of Tennessee \\ R.E. STOLLER \\ Oak Ridge National Laboratory
}

November 2011

Prepared by

OAK RIDGE NATIONAL LABORATORY

Oak Ridge, Tennessee 37831-6283

managed by

UT-BATTELLE, LLC

for the

U.S. DEPARTMENT OF ENERGY

under Contract DE-AC05-00OR22725 


\section{CONTENTS}

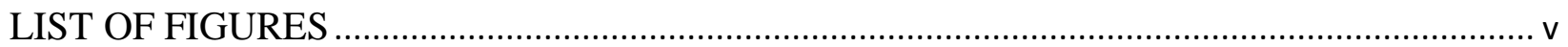

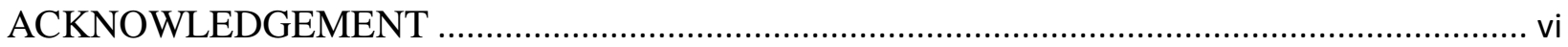

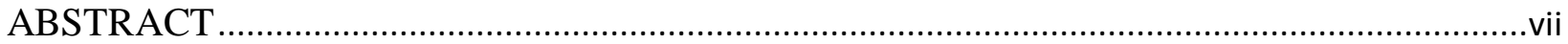

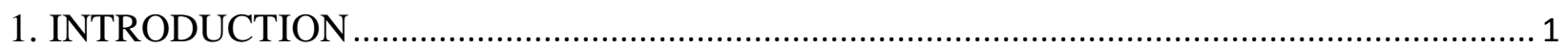

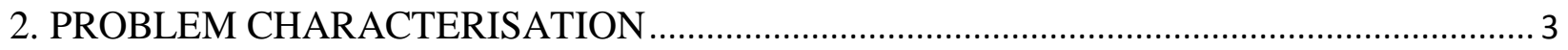

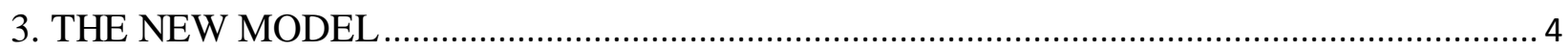

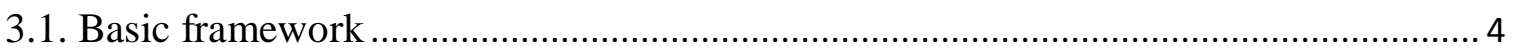

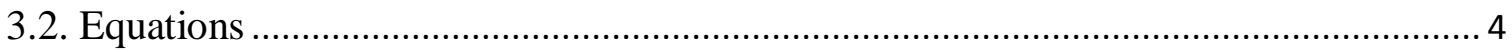

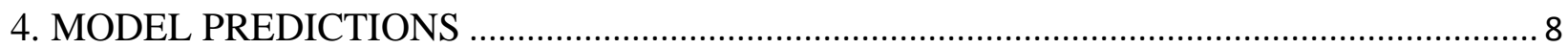

4.1. Stage I of RG in pre-annealed materials …................................................................. 8

4.2. Stage II of RG in pre-annealed materials ............................................................... 8

4.3. Stage III of RG in pre-annealed materials: breakaway grows ........................................ 9

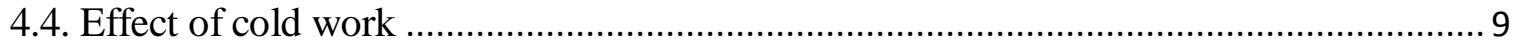

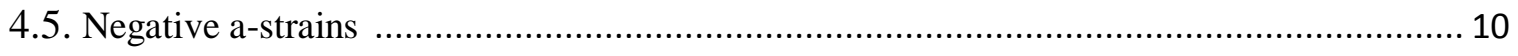

4.6 Coexistence of vacancy and SIA loops ….................................................................. 11

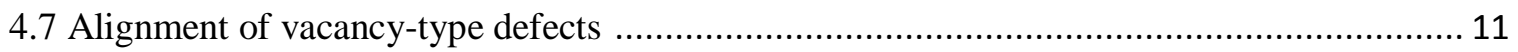

4.8 On the planar alignment of vacancy-type defects in $\mathrm{Zr}$ under electron irradiation ................... 11

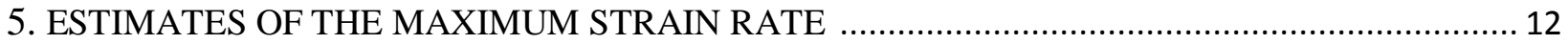

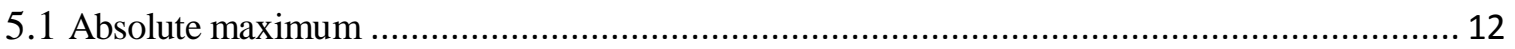

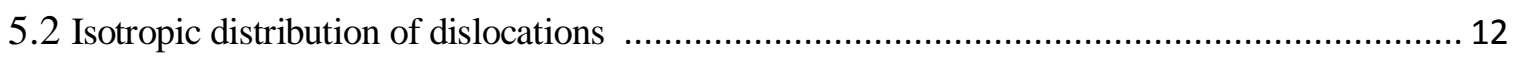

5.3 High strain rates at small density of c-dislocations ......................................................... 13

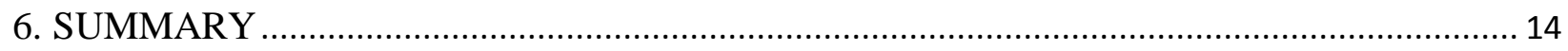

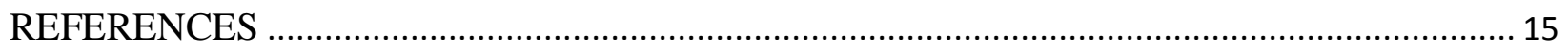

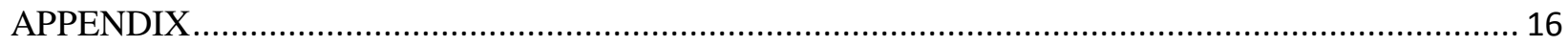

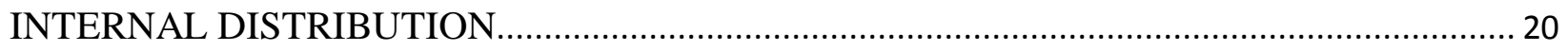

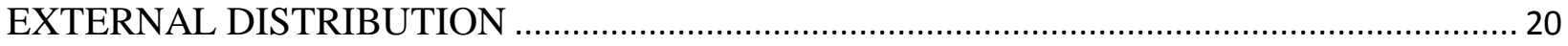




\section{LIST OF FIGURES}

1. Alignment of $c$ loops in bands parallel with the basal planes in neutron-irradiated $\mathrm{Zr}$ (from [16])

2. Alignment of voids in bands parallel to the basal planes in electron-irradiated high purity $\mathrm{Zr}$ (from [12])

3. Dose dependence of growth strain in neutron-irradiated annealed single $\mathrm{Zr}$ crystals (from [23]). Note that the absolute values of the $c$ strain are smaller than that in $a$

4. Main crystallographic directions in hep crystals. ........................................................ 4

5. Dependence of $a$ and $c$ strain rates on fraction of $c$ dislocations...................................... 9

6. Dependence of strain rates in $a$ and $c$ directions on distribution of dislocation Burgers vectors,

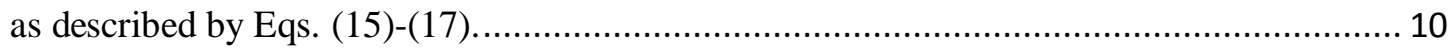

A1. The orientation of prismatic loops near dislocation line ....................................... 16

A2. Interaction energy of 10 SIA cluster with a and c dislocations calculated using Eq.

(A1). The red curves correspond to the case $\alpha=0$, the blue ones to $\beta=\pi / 2 \ldots \ldots 17$

A. 3. Trapping zones of $a$ and $c$ dislocations for an SIA cluster of 10 SIAs 18 


\section{ACKNOWLEDGEMENTS}

This research was supported by the Consortium for Advanced Simulation of Light Water

Reactors (http://www.casl.gov), an Energy Innovation Hub (http://www.energy.gov/hubs) for Modeling and Simulation of Nuclear Reactors under U.S. Department of Energy Contract No. DE-AC05-00OR22725 


\begin{abstract}
A reaction-diffusion model of the radiation growth $(\mathrm{RG})$ of zirconium $(\mathrm{Zr})$ is presented. It takes into account the features of cascade damage: intra-cascade clustering of self-interstitial atoms (SIAs) and their one-dimensional (1-D) diffusion, which occurs under neutron of heavy-ion irradiations. It reproduces all the RG stages observed in neutron-irradiated pre-annealed pure $\mathrm{Zr}$ and $\mathrm{Zr}$ alloys, such as high strain rate at low, strain saturation at intermediate and breakaway growth at relatively high irradiation doses. It accounts for the striking observations of negative strains in prismatic direction and co-existence of vacancy- and SIA-type prismatic loops. It reveals the role of cold work in RG behavior and the reasons for alignment of vacancy-type loops and voids along the basal planes. The maximum possible strain rate is estimated.
\end{abstract}




\section{INTRODUCTION}

The RG of Zr-based materials is one of the main concerns for the safe operation of thermal nuclear reactors, such as PWA and BWA. The experiments demonstrated that the deformation takes place via formation of SIA-type dislocation loops on the prism planes, vacancy loops on the basal planes, and vacancy annihilation at dislocations and grain boundaries.

The first theoretical model of RG was proposed by Buckley about 50 years ago [1] and several other models have been published since then (see, e.g. [2] for a review). A common assumption of the models is that the primary damage is produced in the form of point defects (PDs): single vacancies and SIAs both migrating 3-D. This contradicts molecular dynamics (MD) simulations on intra-cascade clustering of defects and 1-D diffusion of SIA clusters, which are similar in the hcp and cubic metals [3,4]. The work by Holt et al. [5] is the only exception: it takes into account defect clustering but assumes that all the clusters are immobile. Such an unrealistic framework leads to unphysical assumptions when fitting to observations. So, the dislocation bias factor was taken to be equal to $200 \%$ in [5], that is about two orders of magnitude larger than those estimated experimentally in [6,7] and an order of magnitude higher than those calculated using the elasticity theory (see, e.g. [8]). Even with this assumption, the model fails to reproduce many experimental observations.

The situation described is similar to what it was in the research of void swelling in the bcc-and fcc-type metals $\sim 20$ years ago [9], the time when the research of RG and void swelling deviated from each other. Since then the theory of void swelling has made significant progress to account for all observations and become consistent with modeling results (see the Production Bias Model (PBM) in its modern form [6,911]). The distinguishing features of PBM, as compared to older models, are in including (i) the cascadeproduction and (ii) 1-D migration of SIA clusters (small interstitial-type dislocation loops). The PBM succeeds in explaining many striking observations, e.g. the grain boundary effect in swelling and void lattice formation (see [11] for a review).

The displacement cascades in hcp Zr are found to be similar to those in cubic crystals; hence the PBM should provide a realistic framework for the hcp metals. The basal-plane alignment of vacancy loops [4] (Fig. 1) and voids [12] (Fig. 2) observed in hep metals irradiated at low and high temperature, respectively, is analogous to void ordering in cubic metals. This and other similarities give reasons to expect that, with certain modifications for the features of the hcp crystal structure, the PBM will be capable of describing RG. The aim of the present work is to develop such a model.

The paper is organized as follows. In Section 2, the RG is characterized in more details. Sections 3 and 4 describe the background of the new model and give an analysis of the RG. A summary of the work is presented in Section 5. 


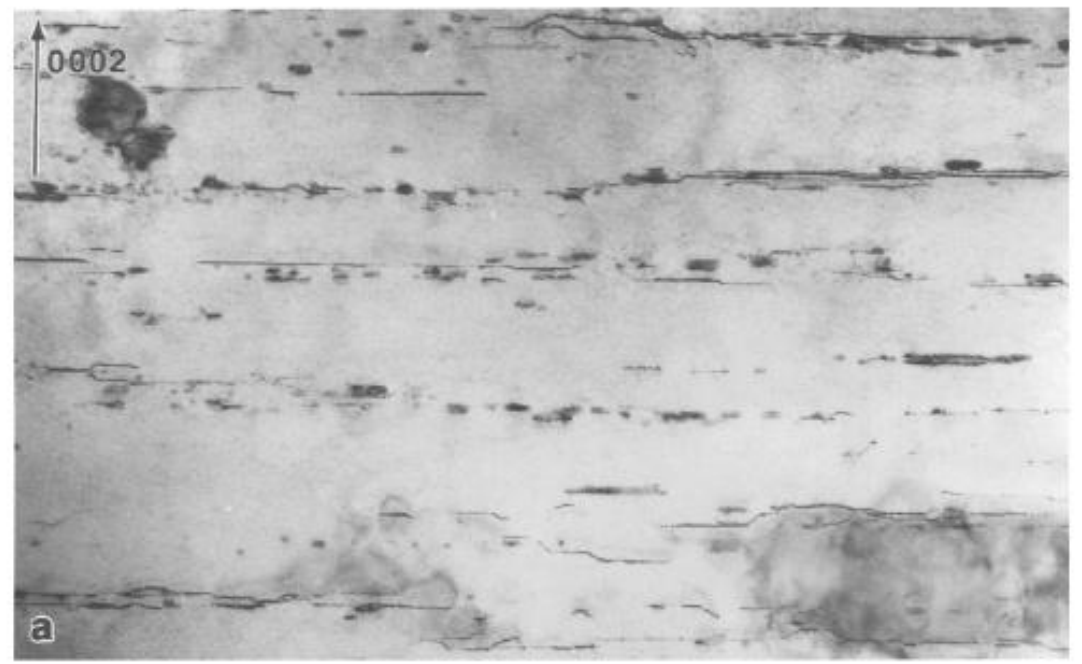

Fig. 1. Alignment of $c$ loops in bands parallel with the basal planes in neutron-irradiated $\mathrm{Zr}$ (from [16]).

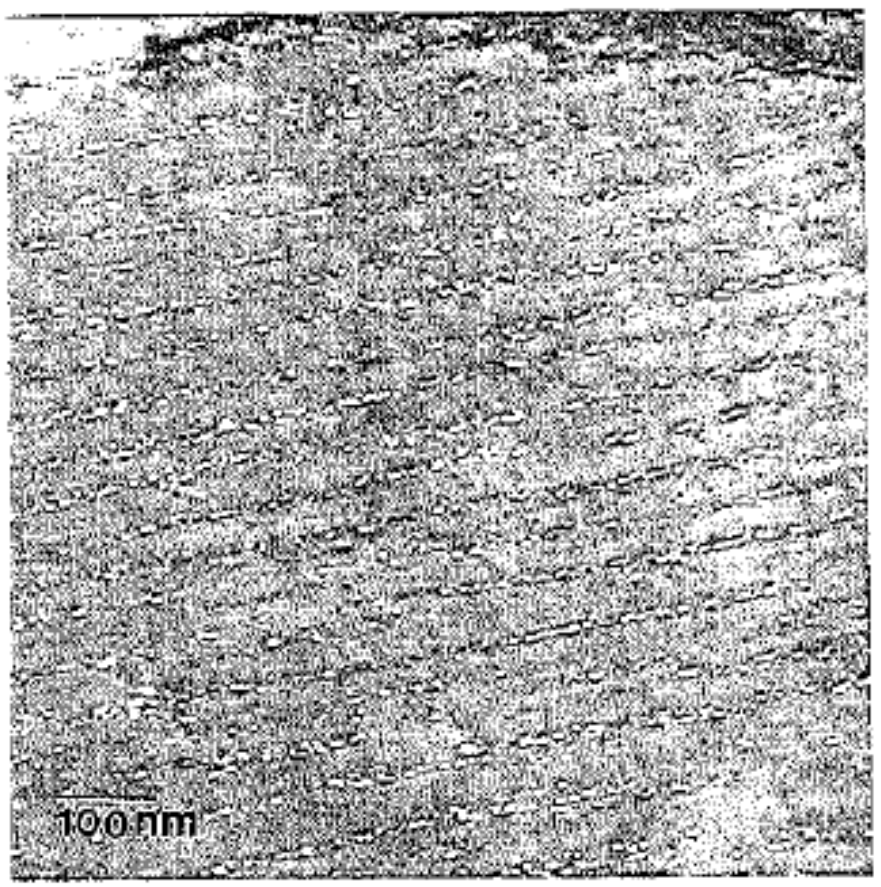

Fig. 2. Alignment of voids in bands parallel to the basal planes in electron-irradiated high purity $\mathrm{Zr}$ (from [12]). 


\section{PROBLEM CHARACTERISATION}

The $\mathrm{RG}$ in $\mathrm{Zr}$ and $\mathrm{Zr}$ alloys at temperatures $T \leq 300^{\circ} \mathrm{C}$ produces an expansion along $a$ - and contraction along $c$-axis. In an annealed $\mathrm{Zr}$, a typical strain behavior is characterized by three distinct stages (see, e.g. [13]). Stage I lasts for 0.1-1.0 displacements per atom (dpa, NRT standard [14]) and exhibits high strain rate. Stage II lasts for $\sim 3 \mathrm{dpa}$ and demonstrates a very low strain rate, which is often interpreted as saturation. The strain rate during the stage III increases with increasing irradiation dose and may reach values as high as $\sim 10^{-3} \mathrm{dpa}^{-1}$, which is about the maximum value observed. This stage is usually referred to as the breakaway growth. Transmission electron microscopy (TEM) reveals formation of prismatic loops with $(1 / 3)\langle 11 \overline{2} 0\rangle$ Burgers vectors during the stages I and II, and $c$-loops during the stage III. The papers published so far have all failed to account for the following observations:

1. Preferential absorption of vacancies by $c$ and $c+a$ dislocations, which have larger Burgers vectors and, hence, higher capture efficiency for single SIAs than $a$ dislocations. This is a major failure of the models where point defects are the only migrating species.

2. High strain rates during stage I and strain saturation during stage II.

3. The breakaway growth and its dose dependence during stage III.

4. Coexistence of vacancy- and SIA-type prismatic loops of similar size and density.

5. Observations of smaller values of the $c$ than $a$ strain and negative $a$ strains.

6. The absolute value of the strain in $c$ direction may be smaller than that in $a$ direction as it can be seen on Fig. 3.

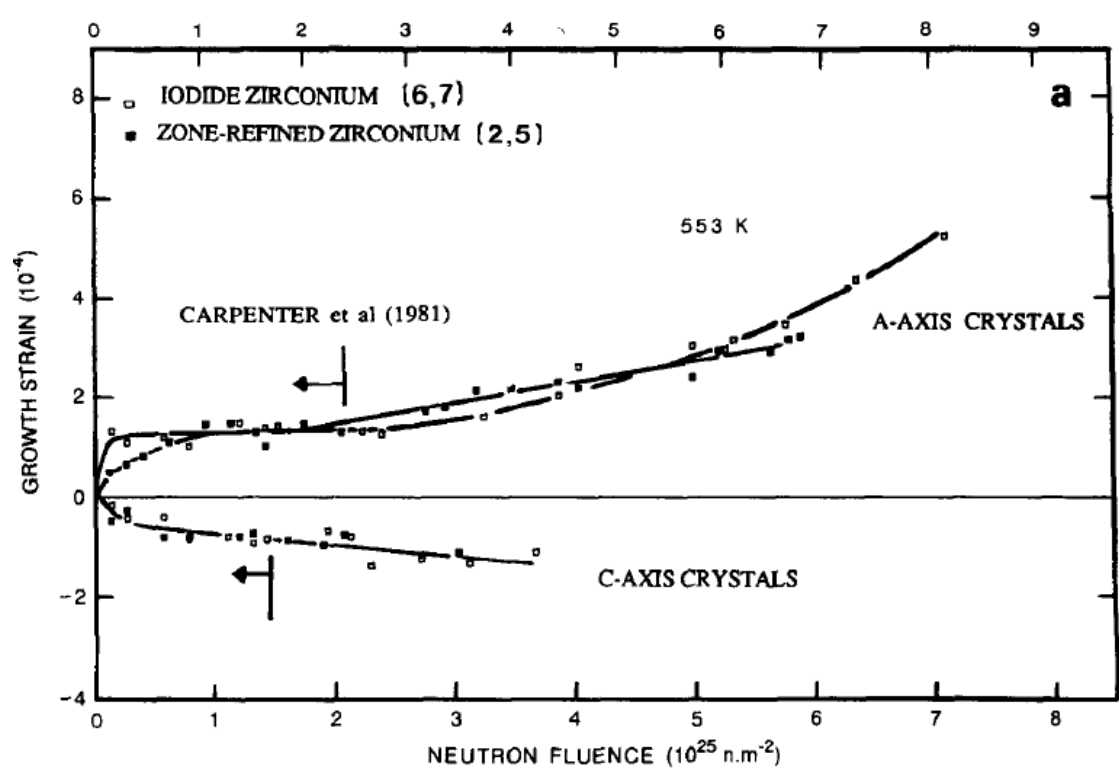

Fig. 3. Dose dependence of growth strain in neutron-irradiated annealed single Zr crystals (from [23]). Note that the absolute values of the $c$ strain are smaller than that in $a$ direction.

In the following we propose a new model which provides explanation for all this. 


\section{THE NEW MODEL}

\subsection{Basic framework}

The new model proposed here is an extension of the PBM (see [11] for a review) to the anisotropic case of hcp crystals. More specifically, the following framework is used:

1. The microstructure consists of $a$ - and $c$-type edge dislocations. Different prismatic directions are treated independently, and the partial densities of dislocations, $\rho_{j}\left(j=\mathbf{a}_{1}, \mathbf{a}_{2}, \mathbf{a}_{3}, \mathbf{c}\right)$, with the Burgers vectors parallel to $\mathbf{a}_{\mathbf{1}}, \mathbf{a}_{\mathbf{2}}$ and $\mathbf{a}_{\mathbf{3}}$ prismatic directions, respectively, may in general be unequal (see Fig. 4).

2. Mobile SIA clusters and PDs are steadily produced in displacement cascades.

3. The PDs execute 3-D random walk, whereas each SIA cluster migrate 1-D along the direction of its Burgers vector, which is one of the close-packed $\langle 11 \overline{2} 0\rangle$ directions along the basal planes.

4. Dislocation bias factor for PDs and mutual recombination of PDs are neglected.

5. No repulsive interactions between defects are considered.

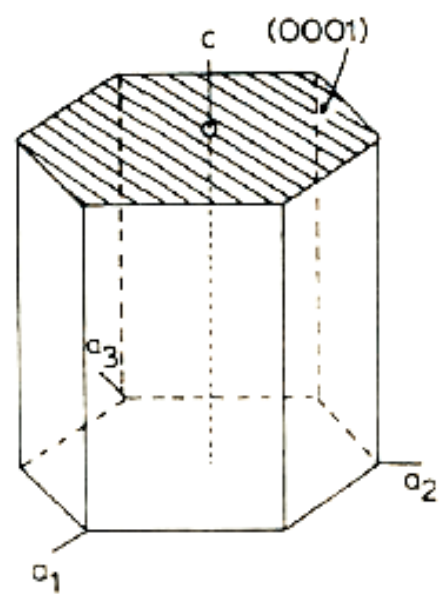

Fig. 4. Main crystallographic directions in hep crystals.

\subsection{Equations}

In the framework of the model formulated, the equations for concentrations, $C$, of single vacancies (subscript v), single SIAs (i) and SIA clusters (cl) are as follows

$$
\begin{array}{cc}
\frac{d C_{\mathrm{v}}}{d t}=G-D_{\mathrm{v}} C_{\mathrm{v}} \sum_{j} \rho_{j}, & \left(j=\mathbf{a}_{1}, \mathbf{a}_{2}, \mathbf{a}_{3}, \mathbf{c}\right) \\
\frac{d C_{\mathrm{i}}}{d t}=G\left(1-\varepsilon_{\mathrm{i}}^{\mathrm{g}}\right)-D_{\mathrm{i}} C_{\mathrm{i}} \sum_{j} \rho_{j}, & \left(j=\mathbf{a}_{1}, \mathbf{a}_{2}, \mathbf{a}_{3}, \mathbf{c}\right) \\
\frac{d C_{\mathrm{cl}}^{m}}{d t}=G \frac{\varepsilon_{\mathrm{i}}^{\mathrm{g}}}{3 n}-D_{\mathrm{cl}} C_{\mathrm{cl}}^{m} k_{m}^{2}, & \left(m=\mathbf{a}_{1}, \mathbf{a}_{2}, \mathbf{a}_{3}\right),
\end{array}
$$


where $G=G_{\mathrm{NRT}}\left(1-\varepsilon_{\mathrm{r}}\right)$ is the defect production rate, $G_{\mathrm{NRT}}$ being the NRT standard rate and $\varepsilon_{\mathrm{r}}$ the fraction of defects recombining in cascades; $\varepsilon_{\mathrm{i}}^{\mathrm{g}}$ is the fraction of SIAs clustered in cascades; $n$ is the mean number of SIAs in a cluster; $D_{\mathrm{v}, \mathrm{i}}$ are the vacancy or SIA diffusion coefficients; $k_{m}^{2}$ is the sink strength for SIA clusters migrating along $m$-th direction. The factor $1 / 3$ on the right-hand side (RHS) of Eq. (3) accounts for the equality of the production rates of SIA clusters in $\mathbf{a}_{1}, \mathbf{a}_{2}$ and $\mathbf{a}_{3}$ directions. The first terms on the RHSs of Eqs. (1)-(3) stand for the production rates of corresponding defects, while the second terms describe their loss at dislocations, including dislocation loops.

The sink strength $k_{m}^{2}$ in Eq. (3) is given by (see, e.g. in [11])

$$
k_{m}^{2}=2\left(\frac{\pi}{2} \sum_{j} \rho_{j} r_{j m}\right)^{2},
$$

where $r_{j m}$ is the capture radius of dislocations with the Burgers vectors along $j$-th direction for the SIA clusters with the Burgers vectors along $m$-th direction. In general, all the interaction radii are nonzero, but the strongest interaction is for dislocations and SIA clusters with parallel and the weakest with perpendicular Burgers vectors. For simplicity, we assume that SIA clusters interact only with dislocations with parallel Burgers vectors:

$$
r_{j m}=r_{0} \delta_{j m}
$$

where $r_{0}$ is the capture radius and $\delta_{j m}$ is the Kronecker delta. Eq. (4) for $k_{m}^{2}$ is then reduced to

$$
k_{m}^{2}=0.5 \pi^{2} r_{0}^{2} \rho_{m}^{2}
$$

The defect steady-state fluxes are found by equating the time derivatives in Eqs. (1)-(3) to zero:

$$
\begin{gathered}
D_{\mathrm{v}} C_{\mathrm{v}}=\frac{G_{\mathrm{NRT}}\left(1-\varepsilon_{\mathrm{r}}\right)}{\rho}, \\
D_{\mathrm{i}} C_{\mathrm{i}}=\frac{G_{\mathrm{NRT}}\left(1-\varepsilon_{\mathrm{r}}\right)\left(1-\varepsilon_{\mathrm{i}}^{\mathrm{g}}\right)}{\rho}, \\
D_{\mathrm{cl}} C_{\mathrm{cl}}^{m}=\frac{2}{3 n} \frac{G_{\mathrm{NRT}}\left(1-\varepsilon_{\mathrm{r}}\right) \varepsilon_{\mathrm{i}}^{\mathrm{g}}}{\pi^{2} r_{0}^{2} \rho_{m}^{2}},
\end{gathered}
$$

where $m=\mathbf{a}_{1}, \mathbf{a}_{2}, \mathbf{a}_{3}$ and $\rho=\sum_{j} \rho_{j}$ is the total dislocation density. The cluster flux in $c$ direction is equal to zero. 
The dislocation climb rates, $V_{m}$, are defined through the net SIA fluxes of interstitials to corresponding dislocations as

$$
V_{j}= \begin{cases}\frac{n}{b_{j}} \frac{D_{\mathrm{cl}} C_{\mathrm{cl}}^{j} k_{j}^{2}}{\rho_{j}}-\frac{D_{\mathrm{v}} C_{\mathrm{v}}-D_{\mathrm{i}} C_{\mathrm{i}}}{b_{j}}, & j=\mathbf{a}_{1}, \mathbf{a}_{2}, \mathbf{a}_{3}, \\ -\frac{1}{b_{j}}\left(D_{\mathrm{v}} C_{\mathrm{v}}-D_{\mathrm{i}} C_{\mathrm{i}}\right), & j=\mathbf{c},\end{cases}
$$

where $\mathbf{b}_{j}$ is the Burgers vector of $j$-th type dislocation. The strain rate, $\varepsilon_{\mathbf{a}}$, due to the climb of prismatic dislocations in a particular a direction is calculated by summing contributions from dislocations with different Burgers vectors $\left(m=\mathbf{a}_{1}, \mathbf{a}_{2}, \mathbf{a}_{3}\right)$ :

$$
\begin{aligned}
\frac{d \varepsilon_{\mathrm{a}}}{d t} & =\sum_{m} \rho_{m} V_{m} b_{m} \cos ^{2} \varphi_{m}= \\
& =\sum_{m}\left[n D_{\mathrm{cl}} C_{\mathrm{cl}}^{m} k_{m}^{2}-\rho_{m}\left(D_{\mathrm{v}} C_{\mathrm{v}}-D_{\mathrm{i}} C_{\mathrm{i}}\right)\right] \cos ^{2} \varphi_{m},
\end{aligned}
$$

where $\varphi_{m}$ is the angle between $\mathbf{a}$ and $\mathbf{b}_{m}$. The strain rate in $c$ direction is given by

$$
\frac{d \varepsilon_{\mathbf{c}}}{d t}=-\rho_{\mathbf{c}}\left(D_{\mathrm{v}} C_{\mathrm{v}}-D_{\mathrm{i}} C_{\mathrm{i}}\right)
$$

By substituting Eqs. (7)-(9) into Eqs. (11) and (12), one finally obtains

$$
\begin{gathered}
\frac{d \varepsilon_{\mathrm{a}}}{d \phi}=\chi \sum_{m}\left(\frac{1}{3}-\frac{\rho_{m}}{\rho}\right) \cos ^{2} \varphi_{m}, \\
\frac{d \varepsilon_{\mathrm{c}}}{d \phi}=-\chi \frac{\rho_{\mathrm{c}}}{\rho},
\end{gathered}
$$

where $\phi=G_{\mathrm{NRT}} t$ is the irradiation dose and $\chi=\left(1-\varepsilon_{\mathrm{r}}\right) \varepsilon_{\mathrm{i}}^{\mathrm{g}}$ is a parameter characterizing the cascade damage. Note that $\chi=0$ for non-cascade conditions, e.g. for irradiation with $\sim \mathrm{MeV}$ electrons, since the model neglects contribution of the point defects to the RG.

In a Cartesian coordinate system where $x$-axis is along $\mathbf{a}_{1}, y$ along $\mathbf{a}_{2}-\mathbf{a}_{3}$, and $z$ along $\mathbf{c}$, Eqs. (13)-(14) take the following form

$$
\frac{d \varepsilon_{x}}{d \phi}=\chi\left(\frac{1}{2}-\frac{\rho_{x}}{\rho}\right)=\chi\left(\frac{\rho_{y}}{\rho}+\frac{\rho_{z}}{\rho}-\frac{1}{2}\right)
$$




$$
\begin{gathered}
\frac{d \varepsilon_{y}}{d \phi}=\chi\left(\frac{1}{2}-\frac{\rho_{y}}{\rho}\right), \\
\frac{d \varepsilon_{z}}{d \phi}=-\chi \frac{\rho_{z}}{\rho},
\end{gathered}
$$

where

$$
\begin{gathered}
\rho_{x}=\rho_{\mathbf{a}_{1}}+\left(\rho_{\mathbf{a}_{2}}+\rho_{\mathbf{a}_{3}}\right) \cos ^{2}(\pi / 3), \\
\rho_{y}=\left(\rho_{\mathbf{a}_{2}}+\rho_{\mathbf{a}_{3}}\right) \cos ^{2}(\pi / 6), \\
\rho_{z}=\rho_{\mathbf{c}} .
\end{gathered}
$$

The second form of Eq. (15) is obtained using the definition for the total dislocation density

$$
\rho=\rho_{x}+\rho_{y}+\rho_{z} \equiv \rho_{\mathbf{a}_{1}}+\rho_{\mathbf{a}_{2}}+\rho_{\mathbf{a}_{3}}+\rho_{\mathbf{c}} .
$$

Note that Eqs. (15)-(17) satisfy the condition of zero total strain rate, independently of the distribution of dislocation Burgers vectors over different directions:

$$
\frac{d \varepsilon_{x}}{d \phi}+\frac{d \varepsilon_{y}}{d \phi}+\frac{d \varepsilon_{z}}{d \phi}=0
$$

which is the volume conservation for pure growth process in the absence of swelling.

Finally, Eqs. (15)-(17) describe the strain rates in a single hcp crystal under cascade damage conditions. The main predictions of the model are described in the next section. 


\section{MODEL PREDICTIONS}

Two basic features of the model follow from Eqs. (15)-(17) and are as follows.

1. The RG is governed by the production of SIA clusters in displacement cascades and their 1-D migration in the close-packed directions along basal planes. Correspondingly, the strain rates are proportional to the parameter $\chi$ is determined by the cascade properties, similarly to void swelling (see, e.g. [9]).

2. Sharing of the vacancy- and SIA- type defects in irradiated hcp crystals is asymmetrical: the vacancies interact with all $a$ - and $c$-type dislocations proportionally to their densities, whereas the SIA clusters are mainly absorbed by $a$ dislocations. The strain rates are determined by corresponding fractions of dislocations: $\rho_{x} / \rho, \rho_{y} / \rho$ and $\rho_{z} / \rho$, rather than by absolute values of dislocation densities.

The model allows explaining, in particular, the following observations.

\subsection{Stage I of $R G$ in pre-annealed materials}

For simplicity, consider isotropic initial distribution of dislocations: $\rho_{x}^{0} / \rho=\rho_{y}^{0} / \rho=\rho_{z}^{0} / \rho=1 / 3$. As can be seen from Eqs. (15)-(17), in this case, the initial strain rates are large: $\chi / 6$ and $-\chi / 3$ for $a$ and $c$ strain rates, respectively, and do not depend on the dislocation densities. This explains the high strain rates observed during stage I.

\subsection{Stage II of RG in pre-annealed materials}

To understand the strain saturation during stage II, one must take into account that with increasing irradiation dose the initial distribution of dislocations is changed due to the cascade production and subsequent growth of $a$ dislocation loops. This leads to an increase of the total dislocation density in $a$ directions: $\rho_{x, y}=\rho_{x, y}^{0}+2 \pi R_{x, y} N_{x, y}$, where $R$ and $N$ are the radius and number density of corresponding loops. Assuming that the distribution of dislocation Burgers vectors along $a$ directions is still isotropic, i.e. $\rho_{x}=\rho_{y}$, one can find from Eqs. (15)-(17) that all the strain rates are directly proportional to the density of $c$ dislocations

$$
\begin{gathered}
\frac{d \varepsilon_{x}}{d \phi}=\frac{d \varepsilon_{y}}{d \phi}=\frac{\chi}{2} \frac{\rho_{\mathrm{z}}}{\rho}, \\
\frac{d \varepsilon_{z}}{d \phi}=-\chi \frac{\rho_{\mathrm{z}}}{\rho} .
\end{gathered}
$$

If $\rho_{\mathrm{z}} / \rho$ is very small, as usually observed in annealed materials and realized if 


$$
2 \pi R_{x, y} N_{x, y}>\rho_{x, y}^{0}
$$

the strain rates may decrease to a level which can be treated as saturation (see arrows on Fig. 5). In other words, the stage II corresponds to a very small but nonzero strain rate.

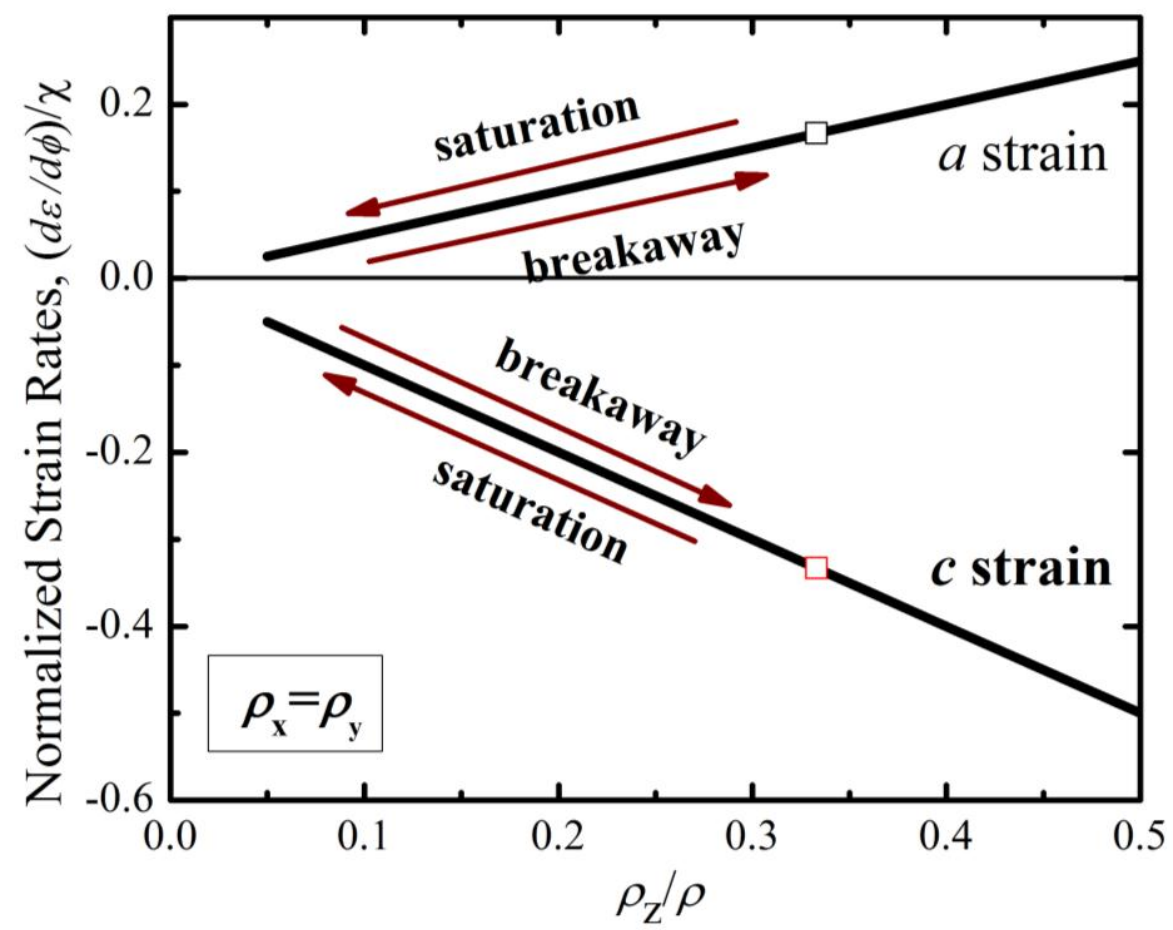

Fig. 5. Dependence of $a$ and $c$ strain rates on fraction of $c$ dislocations.

\subsection{Stage III of RG in pre-annealed materials: breakaway grows}

The occurrence of breakaway growth is known to correlate with the nucleation of $c$-dislocation loops (e.g. [15]). Hence, the total density of $c$ dislocations should increase as $\rho_{\mathrm{z}}=\rho_{\mathrm{z}}^{0}+2 \pi R_{\mathrm{z}} N_{z}$, which will lead to an increase of the ratio $\rho_{\mathrm{z}} / \rho$ and, consequently, of the strain rates, as defined by Eqs. (23) and (24). Note also that similarity of the strain rates during stage I with relatively low and stage III with much higher total dislocation density (including dislocation loops) is explained by the fact that the strain rates are determined by the fractions of dislocations of different Burgers vectors: $\rho_{x} / \rho, \rho_{y} / \rho$ and $\rho_{z} / \rho$, rather than their absolute values.

\subsection{Effect of cold work}


As discussed above, the strain saturation during stage II occurs due to nucleation and growth of $a$ dislocation loops, which increases the total dislocation density in $a$ directions. The condition for occurrence of this stage, Eq. (25), is readily achieved in annealed materials with low initial dislocation density, $\rho_{\mathrm{x}, \mathrm{y}}^{0}$, whereas in cold-worked metals $\rho_{\mathrm{x}, \mathrm{y}}^{0}$ is not low and the condition may not be satisfied, hence the strain rate may not saturate.

\subsection{Negative a-strains}

According to Eqs.(15)-(17), the $c$-strain rate, $d \varepsilon_{z} / d \phi$, is always negative, which is in accordance with observations. In contrast, the strain rate in $a$ direction can be positive or negative depending on the distribution of dislocation Burgers vectors: $\rho_{x}, \rho_{y}$ and $\rho_{z}$, thus explaining observations of negative $a$ strains, e.g. in [13]. The $a$ strain rates in $x$ and $y$ directions may have different signs. This can be seen in Fig. 6 , where the dependence of strain rates on distribution of dislocations with different Burgers vectors are presented, as given by Eqs.(15)-(17).

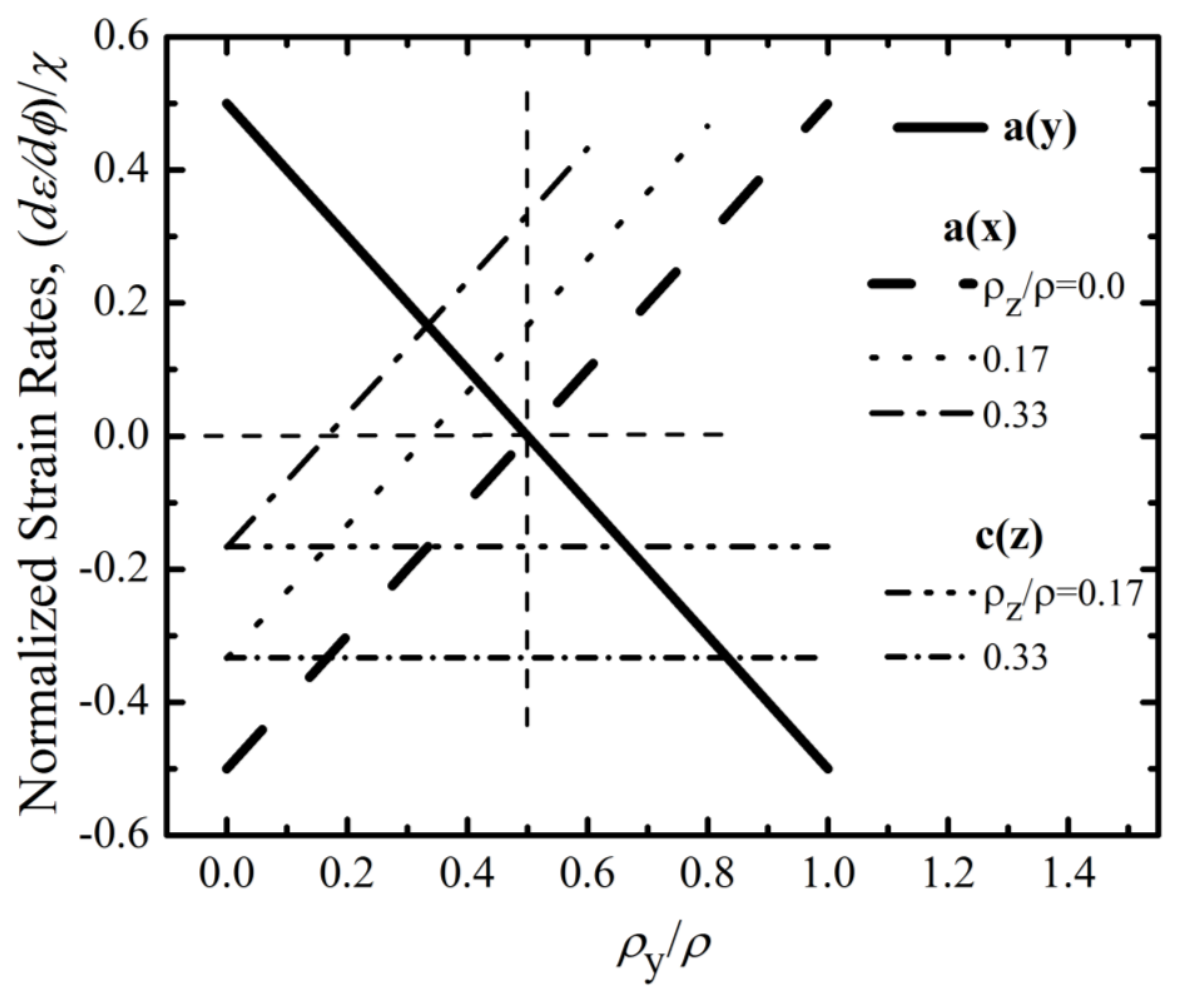

Fig. 6. Dependence of strain rates in $a$ and $c$ directions on distribution of dislocation Burgers vectors, as described by Eqs. (15)-(17). 


\subsection{Coexistence of vacancy and SIA loops}

In older models it was believed that all $a$ directions are equivalent. In this case, the coexistence of vacancy and interstitial loops is problematic. One type of the loops must be kinetically more stable, due to approximately the same net interstitial flux to both types of the loops. In the present model, the $a$ directions may differ because of the non-isotropic distribution of dislocations. This gives possibility to explain observations by assuming that vacancy and SIA loops occupy different equivalent prismatic planes. In this case, obviously, the Burgers vectors of vacancy loops must be along such a direction for which $d \varepsilon_{\mathrm{a}} / d \phi<0$ and vice versa. Experimental data for $a$ strains are not well characterized, as for now, to verify this idea, but necessary experiments can be designed.

\subsection{Alignment of vacancy-type defects}

A planar alignment of the vacancy-type loops and voids in $c$-planes observed in neutron-irradiated hcp metals [16,17] is analogous to void ordering in cubic metals and must have the same origin, which is due to interaction of vacancy defects with the SIA clusters diffusing 1-D along close-packed crystallographic directions (see, e.g. [18]). At high temperature corresponding to the void swelling regime, the void alignment in hcp metals must be driven by the SIA-cluster-void interactions. At low temperature, the alignment of $c$-type vacancy loops must also be due to the interaction with the SIA clusters, but the exact ordering mechanism is not yet clear. There are at least two possibilities: (a) via complete annihilation of unaligned loops or (b) by the loops repulsion by moving SIA clusters. Modeling by MD may clarify the issue.

\subsection{On the planar alignment of vacancy-type defects in $\mathrm{Zr}$ under electron irradiation}

The planar alignment of the vacancy type defects was found in $\mathrm{Zr}$ under irradiation with $1 \mathrm{MeV}$ electrons as well neutrons [12]. This is quite different from cubic crystals, where the void lattices never form under electron irradiation. As yet, the only reasonable explanation proposed for the alignment of vacancy defects is the interaction with interstitial defects with reduced 1- or 2-D migration mode. Since the primary damage in $\mathrm{Zr}$ irradiated with $1 \mathrm{MeV}$ electrons is in the form of single vacancies and SIAs, it is reasonable to expect 1- or 2-D diffusion of single SIAs along basal planes in this metal. A $b$ initio calculations $[19,20]$ predict octahedral to be the most stable SIA configuration in $\mathrm{Zr}$, however, and this should migrate 3-D. The resolution of this discrepancy is important for understanding general physics and constructing interatomic potentials for MD simulations, and is discussed in a forthcoming publication [21]. 


\section{ESTIMATES OF THE MAXIMUM STRAIN RATE}

\subsection{Absolute maximum}

It follows from Eqs. (15)-(17) that the absolute values of strain rates increase with increasing $\rho_{z}$ and take maxima at $\rho_{\mathrm{z}} / \rho \rightarrow 1$ (hence $\rho_{x, y} \rightarrow 0$ ), which are determined by the cascade properties:

$$
\begin{gathered}
\left(\frac{d \varepsilon_{x}}{d \phi}\right)_{\max }=\left(\frac{d \varepsilon_{y}}{d \phi}\right)_{\max }=\frac{\chi}{2}, \\
\left(\frac{d \varepsilon_{z}}{d \phi}\right)_{\max }=-\chi .
\end{gathered}
$$

To make numerical estimates, one needs values for the parameters $\varepsilon_{\mathrm{r}}$ and $\varepsilon_{\mathrm{i}}$. There is no systematic MD and experimental studies of the cascade properties in $\mathrm{Zr}$, so we use data for neutron-irradiated fcc copper: $\varepsilon_{\mathrm{r}}$ $=0.9$ and $\varepsilon_{\mathrm{i}}^{\mathrm{g}}=0.2$ [6], for which $\chi=2 \times 10^{-2}$, since the cascade damage is not drastically sensitive to the type of the lattice. With these data, the maximum strain rates in $a$ and $c$ directions defined by Eqs. (26) and (27) are equal to $10^{-2} \mathrm{dpa}^{-1}$ and $-2 \times 10^{-2} \mathrm{dpa}^{-1}$, respectively. The maximum rates found experimentally are an order

of magnitude smaller, $\sim 10^{-3} \mathrm{dpa}^{-1}$. This large difference is because the condition $\rho_{\mathrm{z}}>>\rho_{\mathrm{x}}, \rho_{\mathrm{y}}$, used to derive Eqs. (26) and (27), is never realized. In fact, the density of $c$-dislocations is always significantly lower than that of $a$-dislocations.

\subsection{Isotropic distribution of dislocations}

For isotropic distribution of dislocation Burgers vectors: $\rho_{\mathrm{x}}=\rho_{\mathrm{y}}=\rho_{\mathrm{z}}$, Eqs. (15)-(17) give

$$
\begin{gathered}
\left(\frac{d \varepsilon_{x}}{d \phi}\right)_{\max }=\left(\frac{d \varepsilon_{y}}{d \phi}\right)_{\max }=\frac{\chi}{6}, \\
\left(\frac{d \varepsilon_{z}}{d \phi}\right)_{\max }=-\frac{\chi}{3}
\end{gathered}
$$

These strain rates are three times smaller than the absolute maximum values given by Eqs. (26) and (27), but still about three times higher than the maximum observed. Usually $\rho_{z}<\rho_{x}, \rho_{y}$, hence the strain rates predicted by the model are close to reality, which is encouraging. Note also that the absorption of the SIA 
clusters by $c$ dislocations is expected to be insignificant and is ignored in the model, but may also contribute to decreasing strain rates.

\subsection{High strain rates at small density of c-dislocations}

The two cases considered above give positive $a$ strain rates. According to Eqs. (15) and (16), this occurs in the region of dislocation densities, defined as

$$
\left(\frac{1}{2}-\frac{\rho_{z}}{\rho}\right) \leq \frac{\rho_{y}}{\rho} \leq \frac{1}{2} .
$$

Outside this region, the strain rates in $x$ and $y$ directions have different signs. In this case, the maximum $a$ strain rates of the order of those given by Eq. (26) may be achieved even at small values of $c$ dislocation fraction. So, if $\rho_{x}, \rho_{z}<<\rho_{y}$ then $d \varepsilon_{x} / d \phi=\chi / 2$ (cf. Eq. (26)), $d \varepsilon_{y} / d \phi=-\chi / 2$, and $d \varepsilon_{z} / d \phi=0$ (see also in Fig. 2). 


\section{SUMMARY}

An RG model of hcp materials irradiated at temperatures below that of the swelling regime $\left(<300^{\circ} \mathrm{C}\right.$ for $\mathrm{Zr}$ ) has been developed, which takes into consideration correct nature of the cascade damage as revealed by the $a b$ initio and MD calculations. In the model, the RG is governed by the production of SIA clusters in displacement cascades and their 1-D migration in the close-packed directions along basal planes, while the main effect comes from asymmetrical sharing of the vacancy- and SIA-type defects: vacancies interact with all dislocations proportionally to their densities, whereas SIA clusters are mainly absorbed by $a$ dislocations. This is because the cascades produce SIA clusters with the Burgers vectors along $a$ directions, hence interact more strongly with $a$ dislocations. This allows all important observations to be accounted for. In particular, the model gives explanation to the following observations:

1. High strain rate at low irradiation doses in pre-annealed materials (stage I of RG).

2. Saturation of strain at intermediate doses in pre-annealed materials (stage II of RG).

3. The breakaway growth at high enough doses in pre-annealed materials (stage III of RG).

4. Coexistence of vacancy- and SIA- type prismatic loops.

5. Higher absolute values of the $c$ strain than those for perfect correspondence to $a$ strain.

6. Negative $a$ strains.

7. Absence of stage II (strain saturation) in materials with high dislocation densities.

8. Alignment of vacancy type defects, dislocation loops and voids, along the basal planes. In addition, the maximum strain rates have been estimated.

The theory proposed resolves the major failure of the older models where the point defects are the only migrating species; namely that the vacancies are absorbed preferentially by $c$ or $c+a$ dislocations, which have larger Burgers vectors and, hence, higher capture efficiency for single SIAs than $a$ dislocations.

The theory proposed predicts positive total prismatic strain, when negative a strain is observed. This result, hence the validity of the model, can be verified experimentally. The success of the model in explaining all main observations indicates that a practical theory of irradiation growth is possible, where the critical processes and, hence, the ways of improving materials properties, may be elucidated. 


\section{REFERENCES}

[1] S.N. Buckley, Properties of Reactor Materials and Effects of Radiation Damage, ed. W.J. Littler (Butterworths, London, 1962) p. 413.

[2] R.A. Holt, J. Nucl. Mater. 372 (2008) 182.

[3] S.J. Wooding, L.M. Howe, F. Gao, A.F. Calder, D.J. Bacon, J. Nucl. Mater. 254 (1998) 191.

[4] N. De Diego, Y.N. Osetsky, D.J. Bacon, In: Proceedings of MRS Fall Meeting; Boston, MA; USA; (2000) p. 200.

[5] R.A. Holt, C .H. Woo, C.K. Chow, J. Nucl. Mater. 205 (1993) 293.

[6] S.I. Golubov, B.N. Singh, H. Trinkaus, Phil. Mag. A81 (2001) 2533.

[7] G.P. Walters, J. Nucl. Mater. 136 (1985) 263.

[8] W.G. Wolfer, Computer-Aided Mater. Des. 14 (2007) 403.

[9] A.V. Barashev, S.I. Golubov, Phil. Mag. 89 (2009) 2833.

[10] B.N. Singh, S.I. Golubov, H. Trinkaus, A. Serra, Yu.N. Osetsky, A.V. Barashev, J. Nucl. Mater. 251 (1997) 107.

[11] S.I. Golubov, A.V. Barashev, R.E. Stoller. "Mean Field Reaction Rate Theory”, In: Encyclopedia of Comprehensive Nuclear Materials, Chapter 29, edited by Rudy Konings, Elsevier Ltd. (2011).

[12] Y. de Carlan, C. Regnard, M. Griffiths, D. Gilbon, C. Lemaignan, ASTM STP 1295 (1996) 638.

[13] M. Griffiths, R.A. Holt, A. Rogerson, J. Nucl. Mater. 225 (1995) 245.

[14] M.J. Norgett, M.T. Robinson, I.M. Torrens, Nucl. Eng. Des. 33 (1975) 50.

[15] R.A. Holt, R.W. Gilbert, J. Nucl. Mater. 116 (1983) 127.

[16] M. Griffiths, J. Nucl. Mater. 159 (1988) 190.

[17] R. Risbet, V. Levy, J. Nucl. Mater. 50 (1974) 116.

[18] A.V. Barashev, S.I. Golubov, Phil. Mag. 90 (2010) 1787.

[19] F. Willaime, J. Nucl. Mater. 323 (2003) 205.

[20] C. Domain, A. Legris, Phil. Mag. 85 (2005) 569.

[21] G.D. Samolyuk, S.I. Golubov, Y.N. Osetsky, R.E. Stoller, DFT study revises interstitial configurations in hcp Zr, ORNL TM-2011/-516.

[22] M.J. Makin, Phil. Mag. 106 (1964) 695.

[23] G.J.C. Carpenter, R.A. Murgatroyd, A. Rogerson, J.F. Watters, J. Nucl. Mater. 101 (1981) 28. 


\section{APPENDIX}

The interaction between a dislocation loop and an edge dislocation depends strongly on their mutual orientation. This was analyzed by Makin [22] using infinitesimal loop approximation in the framework of the isotropic elasticity theory. The corresponding interaction energy can readily be obtained with the aid of Eqs. (1) in [22] for the components of the stress tensor, $\sigma_{i j}$, as

$$
E=A \sum_{i, j} \sigma_{i j} b_{i} n_{j},
$$

where $b_{i}$ is the component of the cluster Burgers vector on $j$ direction and $A n_{j}$ is the area of the loop resolved onto a plane perpendicular to the $i$ direction. The result is as follows.

Consider a Cartesian coordinate system with $x_{1}, x_{2}$ and $x_{3}$ axes and a straight edge dislocation with its line along $x_{3}$ direction (line sense) and the Burgers vector along $x_{1}$ (see Fig. A1), and distinguish two cases. The first case represents an $a$-type dislocation with the Burgers vector $b_{\mathrm{a}}$; the SIA cluster moves in the plane perpendicular to $x_{3}$ direction, hence containing $x_{1}$ and $x_{2}$ vectors and its Burgers vector is at an angle $\alpha$ to the dislocation Burgers vector and $x_{1}$. The interaction energy in this case is defined by the following equation:

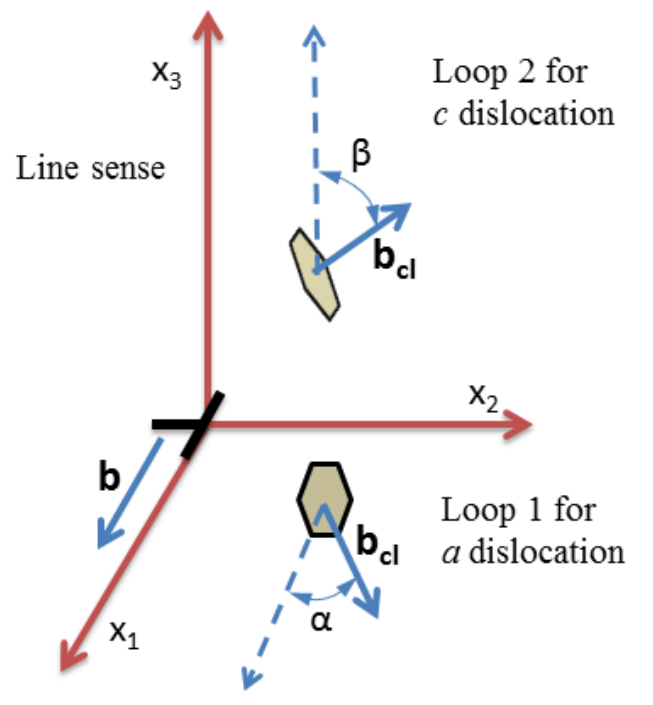

Fig. A1. The orientation of prismatic loops near dislocation line.

$$
E_{\mathrm{a}}=\frac{E_{0} b_{\mathrm{a}} n}{\left(x_{1}^{2}+x_{2}^{2}\right)^{2}}\left[-x_{2}\left(3 x_{1}^{2}+x_{2}^{2}\right) \cos ^{2} \alpha+2 x_{1}\left(x_{1}^{2}-x_{2}^{2}\right) \cos \alpha \sin \alpha+x_{2}\left(x_{1}^{2}-x_{2}^{2}\right) \sin ^{2} \alpha\right],
$$


where $E_{0}=\mu \Omega / 2 \pi(1-v) ; \mu$ is the shear modulus, $v$ is the Poisson ratio, $\Omega$ is the atomic volume, and $n$ is the number of SIAs in the loop, which enters via the relationship $A b=\Omega n$.

The second case corresponds to the $c$-type dislocation with the Burgers vector $b_{\mathrm{c}}$. In this case, the SIA cluster moves in the plane perpendicular to $x_{1}$ direction, hence containing $x_{3}$ and $x_{2}$ vectors, and its Burgers vector is at an angle $\beta$ to the dislocation line and $x_{3}$. The interaction energy in this case is given by

$$
E_{\mathrm{c}}=\frac{E_{0} b_{\mathrm{c}} n}{\left(x_{1}^{2}+x_{2}^{2}\right)^{2}}\left[x_{2}\left(x_{1}^{2}-x_{2}^{2}\right) \sin ^{2} \beta-2 v x_{2}\left(x_{1}^{2}+x_{2}^{2}\right) \cos ^{2} \beta\right] .
$$

The interaction energy of $a$ and $c$ dislocations with a 10 SIA cluster at $573 \mathrm{~K}$ is presented on Fig. A2 as a function of the distance along the cluster Burgers vector direction for different impact factors: the closest distance between the cluster and dislocation line. The red curves on this figure correspond to $\alpha=0$ in Eq. (A1), when the Burgers vectors of an SIA cluster and an $a$ dislocation are parallel to each other, hence are described by the following equation:

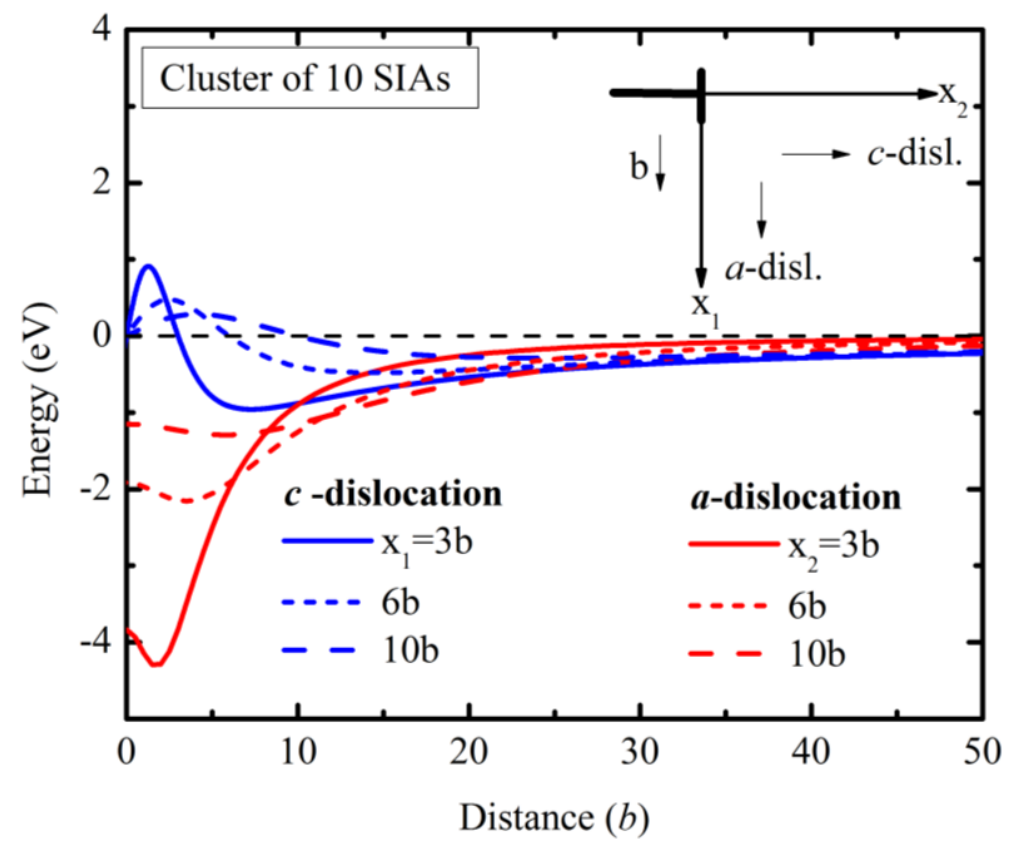

Fig. A2. Interaction energy of 10 SIA cluster with $a$ and $c$ dislocations calculated using Eq. (A1). The red curves correspond to the case $\alpha=0$, the blue ones to $\beta=\pi / 2$. 


$$
E_{\mathrm{a}}(\alpha=0)=-E_{0} b_{\mathrm{a}} n \frac{x_{2}\left(3 x_{1}^{2}+x_{2}^{2}\right)}{\left(x_{1}^{2}+x_{2}^{2}\right)^{2}} .
$$

The blue curves correspond to the case $\beta=\pi / 2$ in Eq. (A2) for the cluster interaction with $c$ dislocation:

$$
E_{\mathrm{c}}\left(\beta=\frac{\pi}{2}\right)=-E_{0} b_{\mathrm{cd}} n \frac{x_{2}\left(x_{2}^{2}-x_{1}^{2}\right)}{\left(x_{1}^{2}+x_{2}^{2}\right)^{2}} .
$$

The calculations were performed with $\mu=33 \mathrm{GPa}, \nu=0.34, \Omega=2.58 \times 10^{-29} \mathrm{~m}^{-3}$, for which $E_{0}=1.15 \mathrm{eV}$.

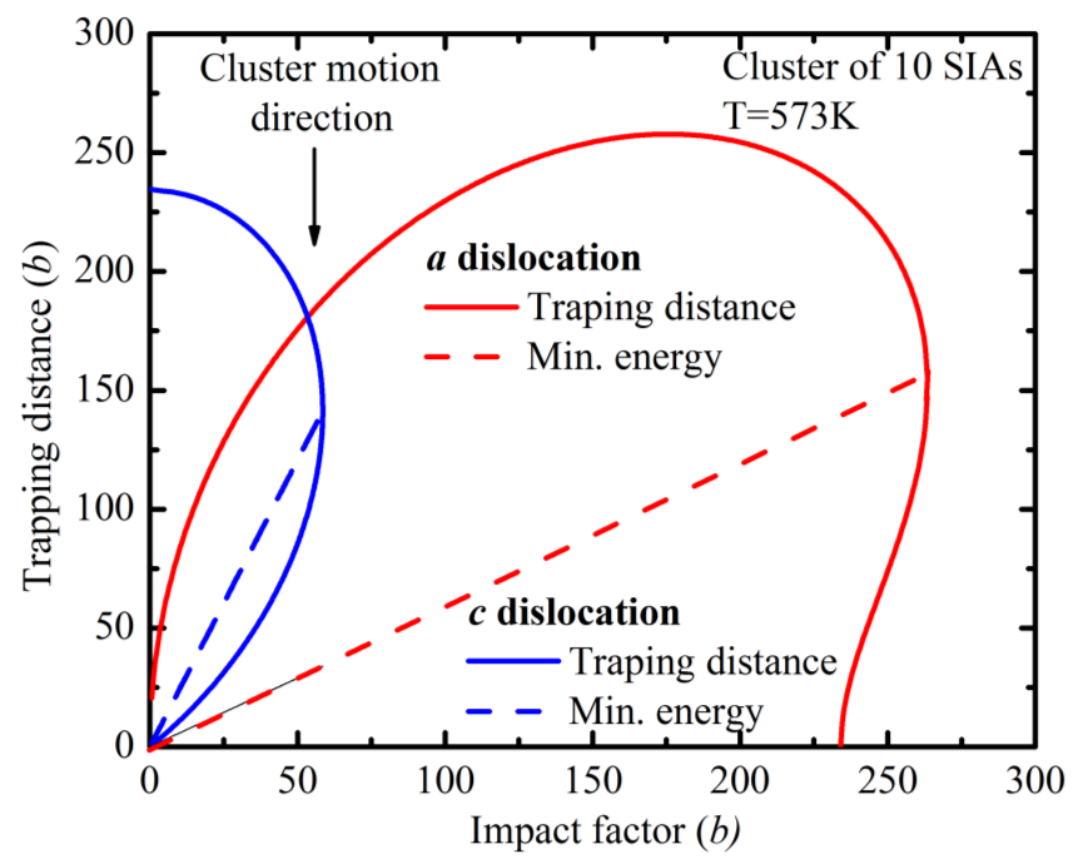

Fig. A3. Trapping zones of $a$ and $c$ dislocations for an SIA cluster of 10 SIAs.

Fig. A3 shows the trapping zone boundaries of $a$ and $c$ dislocations for a 10-SIA cluster at 573K for the same cases as on Fig. A2 (i.e. $\alpha=0$ and $\beta=\pi / 2$ ). These are defined by the equality of the binding energy and the thermal energy $k_{\mathrm{B}} T$ ( $k_{\mathrm{B}}$ being the Boltzmann constant, $T$ the absolute temperature): $E_{\mathrm{a}, \mathrm{c}}=k_{\mathrm{B}} T$, and are described by the following equations for $a$ and $c$ dislocations, respectively:

$$
\begin{gathered}
x_{1}=x_{2} \sqrt{\left(3 f_{\mathrm{a}}-1\right) \pm \sqrt{\left(3 f_{\mathrm{a}}-1\right)^{2}-\left(1-f_{\mathrm{a}}\right)}}, \\
x_{1}=x_{2} \sqrt{\left(f_{\mathrm{c}}-1\right) \pm \sqrt{\left(f_{\mathrm{c}}-1\right)^{2}-\left(1+f_{\mathrm{c}}\right)}},
\end{gathered}
$$

where $f_{\mathrm{a}}=\tilde{E} b_{\mathrm{a}} / x_{2}, f_{\mathrm{c}}=\tilde{E} b_{\mathrm{c}} / x_{2}$, and $\tilde{E}=n E_{0} / 2 k_{\mathrm{B}} T$. The dashed lines on Fig. A3 show the positions of the minimum interaction energy (maximum attraction): 


$$
\begin{gathered}
x_{1}=x_{2} / \sqrt{3 \tilde{E}}, \\
x_{1}=x_{2} \sqrt{3 \tilde{E}},
\end{gathered}
$$

for $a$ and $c$ dislocations, respectively.

As can be seen from the figures, there is a qualitative difference between the cluster interaction with $a$ and $c$ dislocations: in the first case, the clusters are attracted at any distance, whereas, in the latter case, there is a repulsion area around the dislocation core and attraction beyond this area. Hence, the absorption of clusters by $c$ dislocations should probably not happen. Also, as can be seen in Fig. A3, the clusters should spend much of their time near $c$ as well as $a$ dislocations in the areas, where $-E_{\mathrm{a}, \mathrm{c}}>k_{\mathrm{B}} T$. So, one may expect that the decoration of $c$ dislocations, as well as $a$ dislocations, with the SIA clusters should takes place. 
ORNL/TM-2011/473

\section{INTERNAL DISTRIBUTION}

1. S. J. Zinkle, zinklesj@ornl.gov

2. M. Stocks, stocksgm@ornl.gov

3. J. R. Morris, morrisj@ornl.gov

4. D. N. Nicholson, nicholsondm@ornl.gov

5. D.B Kothe, kothe@ornl.gov

6. G.I. Ice, icege@ornl.gov

7. B. Wirth, wirthbd@ornl.gov

8. ORNL office of Technical Information

\section{EXTERNAL DISTRIBUTION}

9. C.A. English, Nexia Solutions, UK, colin.english@materials.ox.ac.uk, colin.a.english@nexiasolutions.com

10. D.J. Bacon, The University of Liverpool, Department of Engineering, Brownlow Hill, Liverpool, L69 3GH, UK, djbacon@liverpool.ac.uk

11. A. Serra, Universitat Politècnica de Catalunya, Dept. Matemàtica Aplicada III, ETSECCPB and CRNE, 08034, Barcelona, Spain, a.serra@upc.edu

12. Malcolm Griffiths, Atomic Energy of Canada Limited, Chalk Rover Laboratories, Chalk River, Ontario, Canada, griffithsm@aecl.ca

13. R.A. Holt, Department of Mechanical and Materials Engineering, Queen's University at Kingston, Ont., Canada K7L 1W9, holt@me.queensu.ca

14. Simon R. Phillpot, Department of Materials Science and Engineering, University of Florida, 100 Rhines Hall, PO Box 116400, Gainesville FL, 32611-6400, sphil@mse.ufl.edu

15. Stephen M. Foiles, Computational Materials Science and Engineering Dept., Sandia National Laboratories, Albuquerque, NM 87185-1411, foiles@ sandia.gov

16. Donald Brenner, Department of Material Science and Engineering, North Carolina State University, Raleigh, NC, 276957907, brenner@ncsu.edu

17. Carlos N. Tome, MST-8, MS G755, Los Alamos National Laboratory, Los Alamos, New Mexico 87545, USA, tome@lanl.gov

18. B.P. Uberuaga, Materials Science and Technology Division, Los Alamos National Laboratory, Los Alamos, New Mexico 87545, USA, blas@lanl.gov

19. Arthur F. Voter, Theoretical Division, Los Alamos National Laboratory, Los Alamos, New Mexico 87545, USA, afv@lanl.gov

20. C. Domain, EDF-R\&D Department MMC, Les Renardieres, F-77818 Moret sur Loint Cedex, France, christophe.domain@edf.fr

21. F. Willaime, Service de Recherches de Metallurgie Physique, DMN-SRMP, CEA Saclay, 91191 Gif-sur-Yvette, France, francois.willaime@cea.fr

22. Ishino Shiori, Editor of Journal of Nuclear Materials, ishino@k9.dion.ne.jp 
23. H. Kaburaki, Nuclear Science and Engineering Directorate: Japan Atomic Energy Agency, Japan, kaburaki.hideo@jaea.go.jp

24. S.L. Dudarev, EURATOM/UKAEA Fusion Association, Culham Science Centre, Oxfordshire OX14 3DB, UK, Sergei.Dudarev@UKAEA.org.uk

25. A. Legris, Bâtiment C6 - 225, Unité Matériaux et Transformations, Bât. C6, Université Lille1, 59655 Villeneuve d'Ascq, France, alexandre.legris@univ-lille1.fr,

26. D. Nguyen-Manh, Materials Modeling Laboratory, Department of Materials, University of Oxford, Parks Road, Oxford OX1 3PH, manh.nguyen@ materials.ox.ac.uk

27. M.W. Finnis, Imperial College London, Department of Materials, 105 Royal School of Mines, South Kensington Campus, m.finnis@imperial.ac.uk

28. A. Sutton, Imperial College London, Department of Materials, 105 Royal School of Mines, South Kensington Campus, a.sutton@imperial.ac.uk

29. F. Soisson, Service de Recherches de Metallurgie Physique, DMN-SRMP, CEA Saclay, 91191 Gif-sur-Yvette, France, Frederic.soisson@cea.fr

30. Sidney Yip, Department of Nuclear Science and Engineering, Massachusetts Institute of Technology, 77 Massachusetts Avenue, 24-107, Cambridge, MA 02139, syip@MIT.EDU

31. C.R Stanek, Los Alamos National Laboratory, Los Alamos, New Mexico 87545, USA, stanek@lanl.gov

32. G.S. Was, University of Michigan, Nuclear Engineering and Radiological Sciences, Cooley Building, Ann Ardor, MI 48109-2104, USA, gsw@umich. Edu

33. M.I. Mendelev, Materials and Engineering Physics, Ames Laboratory, US DOE, 207 Metals Development, Ames, IA, USA, mendelev@ameslab.gov

34. G.J. Ackland, 2502 JCMB, ICMCS School of Physics, University of Edinburgh, Edinburgh EH9 3JZ, G.J.Ackland@ed.ac.uk

35. D. Terentyev, Nuclear Materials Science, SCK-CEN, Boeretang 200, B-2400 Mol, Belgium, dterenty@sckcen.be

36. P. Vladimirov, Karlsruhe Institute of Technology (KIT), Institute for Applied Materials Applied Materials Physics, Hermann-von-Helmholtz-Platz 1 IAM-AWP/MW, 76344 Eggenstein-Leopoldshafen, Germany, Pavel.Vladimirov@kit.edu 